\title{
BERBERINE INDUCES AUTOPHAGY, APOPTOSIS, AND MODULATES MIR-155 IN HEAD AND NECK SQUAMOUS CARCINOMA CELLS
}

\author{
BINBIN ZHANG ${ }^{1}$, JINGCHUAN $\mathrm{HE}^{2}$ and KAI XUE ${ }^{1 *}$
}

${ }^{1}$ Department of Neurosurgery, Tianjin Huanhu Hospital, Tianjin, China, 300060

${ }^{2}$ Department of Otorhinolaryngology, Tianjin Huanhu Hospital, Tianjin, China, 300060

\begin{abstract}
Berberine (BBR) an active natural plant alkaloid extracted from Coptidis rhizoma, displays potent anticancer activity over a variety of cancer cell lines. The cytotoxic activity of BBR in cancer cells is attributed to persuade, programmed cell death characterized by the release of cytochrome $\mathrm{c}$, accompanied by activation of caspase- 3 and caspase-9. In the present study, we evaluated BBR significantly reduces the cell viability and clonogenic property of head and neck squamous carcinoma (HNSC) cells. Our results revealed that BBR simultaneously induces apoptosis and autophagy in HNSC cells. Mechanistically, BBR induces autophagy in HNSC cells which were confirmed by acridine orange (AO) staining by visualization of prominent orange-red color acidic autophagosomes in the cytoplasm. However, immunoblotting shows the steady conversion of MAP-LC3I to LC-3II with concomitant degradation of autophagy substrate protein SQSTM1/p62. Annexin V FITC staining analysis by flow cytometry revealed a significant induction of apoptosis at higher doses of BBR. Furthermore, the immunoblotting analysis revealed a prominent cleavage of proapoptotic proteins procaspase3 and PARP1 at higher doses of BBR. Additionally, we found significant upregulation and downregulation of tumor suppressor microRNA-155 (miR-155) and oncogenic miR-21 respectively, when HNSC cells were exposed to higher doses of BBR. In conclusion, these results demonstrate that BBR exhibits a significant antiproliferative effect with the simultaneous induction of autophagy and apoptosis and modulates miRNA expression in HNSC cells.
\end{abstract}

Keywords: berberine, autophagy, apoptosis, miR-155, head and neck cancer cells

Head and neck cancer (HNC) ranks sixth most common cancer across the globe and adds approximately 0.5 million new cases annually (1). The major oncogenic drivers are high intake of alcohol, long-term use of tobacco, and high-risk infectionsassociated Human Papilloma Virus (2). The therapeutic modalities for HNC are surgery followed by radio and chemotherapy. However, the outcome of these therapeutic modalities is dismal which drastically reduces the quality of life and five year survival time (2). Owing to very high heterogeneity in HNSC cells, the current regiment of chemotherapeutic drugs against HNSC is not significantly improving the outcome of the disease (2). Intriguingly, these drugs have a range of adverse side effects, such as immune-suppression, myelosuppression, gastrointestinal distress, and infertility. One of the major debacles of current chemotherapy is the development of drug resistance which becomes a major cause of treatment failure (3).
Thus, identification of novel and effective treatment strategies that might be more effective (hit multiple target sites), with fewer side effects and sensitize drug-resistant cells. Owing to have fewer toxicity issues to normal cells and hit multiple targets in malignant cells, natural compounds obtained from plant sources act as an excellent chemopreventive agent with a safe toxicity profile.

Micro RNAs (miRNAs) are small single, noncoding RNA molecules, evolutionary conserved comprised of about 19-23 nucleotides. The primary function of miRNAs is the modulation of transcription by binding to mRNAs 3-untranslated region (4). MiRNAs modulates the entire pathophysiological processes including proliferation, invasion, angiogenesis, metastasis, apoptosis, and differentiation in diseases like cancer (5). Various types of tumors have altered the expression of miRNAs. During the development of tumorigenesis, some miRNAs are downregulated or upregulated (6).

\footnotetext{
* Corresponding author: e-mail: tracyhunteruwmsmz@yahoo.com
} 
Moreover, recent reports suggest that various chemotherapeutic drugs regulating epigenetics thereby modulate miRNA expression by downregulating oncogenic miRNAs (7). Owing to have deregulated expression in various types of cancers, miRNAs are the appealing targets in both cancer diagnostics and therapeutics.

BBR, a phytochemical derived from Coptidis rhizome, was exclusively studied over the last decade (8). Its effectiveness as an anti-inflammatory, anti-depressor, anti-bacterial, anti-viral, anti-diabetic, and more had put it as a center of research, especially as an anti-cancer agent (8). This inhibitory effect of BBR was elucidated by the antiproliferative effects on different types of cancers including head and neck, lung, breast, glioblastoma, etc., by targeting different signaling pathways $(9,10)$. Recently the autophagy induced cell death was demonstrated as a second type of programmed cell death, in different types of tumors (11). In recent reports from various scientific outcomes revealed that BBR persuades tumor cell killing by activating apoptosis and autophagy (12).

Autophagy is the basic catabolic mechanism that involves the degradation of unnecessary or dysfunctional cellular components with the help of lysosomes. This results in the recycling of cellular components for metabolic energy to regulate cellular homeostasis, especially during stress conditions (12-14). In recent reports, autophagy has been demonstrated to play key roles in numerous physiological and pathological processes, like cell proliferation, differentiation, and metabolism (13). In this study, we demonstrated that BBR exhibits antiproliferative activity by simultaneously inducing autophagy and apoptotic cell death in HNSC cells. Additionally, we demonstrate BBR modulates the expression of miRNAs by upregulates the expressions of miR-155 and downregulates the expressions of miR-21 in a dose-dependent manner.

\section{MATERIALS AND METHODS}

\section{Cell culture and treatments}

HNSC cells (SSC-15 \& SSC-4) and normal transformed cell line NBL-4 were procured from the American Type Culture Collection (ATCC). The cells were maintained in a proper culture medium as the instructions provided on the ATCC datasheet. Briefly, HNSC cells were maintained in the required medium supplemented by $10 \%$ fetal bovine serum (FBS). Culturing cells were incubated at $37^{\circ} \mathrm{C}$ in the $\mathrm{CO}_{2}$ incubator contains $5 \% \mathrm{CO}_{2}$. Cells were periodically checked for mycoplasma contamination.

\section{Chemicals, reagents, and antibodies}

BBR, 3-(4,5-dimethylthiazol-2-yl)-2, 5-diphenyltetrazolium bromide (MTT), phenylmethylsulphonyl fluoride (PMSF), acridine orange (AO), crystal violet, propidium iodide (PI), and DMSO were purchased from Sigma. However, all antibodies that were procured from cell signaling technology are $\beta$ actin, MAP-LC-3I/II, SQSTM1/P62, Beclin 1, PARP 1, and Caspase-3. The secondary anti-mouse IgG and anti-rabbit IgG-tagged with horseradish peroxidase (HRP) antibodies were ordered and purchased from Santacruz Biotechnology.

\section{Cell proliferation/viability assay}

The proliferation of cells was analyzed by MTT assay as per the standard protocol (15). Briefly, HNSC cells (SSC-15 and SSC-4) were processed for trypsinization and $2 \times 10^{3}$ cells were seeded per well in 96-well plates. The seeded cells were attached to the surface overnight and exposed to varying doses of BBR $(100 \mathrm{nM}-1000 \mu \mathrm{M})$ along with control DMSO for $24 \mathrm{~h}$ and incubated in a $5 \%$ $\mathrm{CO}_{2}$ incubator. Subsequently, after the completion of the time point, cells were saturated with MTT dye $(2.5 \mathrm{mg} / \mathrm{mL})$ for $3-4 \mathrm{~h}$ at $37^{\circ} \mathrm{C}$. The formazan crystals formed were solubilized in DMSO, mixed properly by gentle vortex for $15 \mathrm{~min}$, and the optical density was measured at $570 \mathrm{~nm}$ by multi-plate reader. The percentage of cell proliferation was analyzed as the percent cell viability of treated cells compared with the control DMSO group.

\section{Colony formation assay}

SSC- 15 cells at a density of $1 \times 10^{3}$ were plated in 6-well plates as per standard protocol (16). The cells were exposed to varying concentrations of BBR $(200,250,300 \mu \mathrm{M})$ along with control DMSO and were maintained in $5 \% \mathrm{CO}_{2}$ incubator at $37^{\circ} \mathrm{C}$ for $24 \mathrm{~h}$. After the completion of the time point, cells are washed with ice-cold PBS and fixed in pre-cold methanol for 20 minutes. After fixation cells were stained with $0.5 \%$ crystal violet solution for $15 \mathrm{~min}$, wash the cells with distilled water and air dry. The colonies stained in each well were counted and photographed with an Olympus C-7070 microscope.

\section{Immunoblotting}

After overnight plating of SSC-15 cells at a density of $50 \times 10^{5}$ cells/well as per standard protocol (17) in a $5 \% \mathrm{CO}_{2}$ humidified incubator at $37^{\circ} \mathrm{C}$. After adhering to the bottom surface of the well, SSC cells were exposed to varying concentrations of BBR (200, 250, $300 \mu \mathrm{M})$ along with the DMSO vehicle. After 24 h, SSC-15 cells were washed with 
pre-cooled PBS and processed for lysis with cell lysis buffer. The lysis solution obtained from cells was collected and processed at $4^{\circ} \mathrm{C}$ for centrifugation at a speed of $12000 \mathrm{~g}$ for $15 \mathrm{~min}$. Supernatants collected from centrifugation in a separate autoclaved micro-tube were subjected to the Bradford method of protein estimation. The $15 \mu \mathrm{g}$ of protein concentration was calculated from each treated sample and were loaded in the wells of the gel formed of SDS-PAGE. After resolving properly, SDS-PAGE gel was subjected to transfer onto the PVDF membrane, allowed it to incubate in fat-free milk $(5 \%)$ blocking solution dissolved in TBST. After blocking nonspecific protein binding sites with a blocking solution, the PVDF membrane is incubated and probed with the desired antibodies (1: 1000 dilutions) for at least $4 \mathrm{~h}$ when incubated at room temperature or $4^{\circ} \mathrm{C}$ overnight. The PVDF membrane is gently washed with TBST buffer three times. The pre-incubated membrane with the primary antibody is probed with a horseradish peroxidase-labeled secondary antibody. Immunosubstance was spotted by enhanced chemiluminescence (ECL) plus.

\section{Detection of acidic vesicular organelles}

Detection and quantification of acidic vesicular organelles by AO dye were performed using ?uorescence microscopy as described previously (14). Under normal conditions AO stains DNA and cytoplasm and appears green in color, however, in acidic compartments like autophagosomes, AO acts as an anisotropic ?uorescent dye and ?uoresces bright red color in these compartments. Briefly, SSC-15 cells were exposed to different concentrations of BBR $(200,250,300 \mu \mathrm{M})$ along with control DMSO for 24 $\mathrm{h}$ in $5 \%$ humidified $\mathrm{CO}_{2}$ incubator at $37^{\circ} \mathrm{C}$. After the completion of $24 \mathrm{~h}, \mathrm{SSC}-15$ cells were processed with $\mathrm{AO}$ stain $(1 \mathrm{mg} / \mathrm{mL})$ for $15 \mathrm{~min}$ and wash the cells with PBS. The detection of AVOs which appears bright red color under fluorescence was analyzed by fluorescence microscope LSM-510 (Carl Zeiss, Germany).

\section{Apoptosis detection using flow cytometry}

Detection of apoptosis was performed by using staining annexin V-FITC apoptotic detection kit and quantification of apoptotic cells was performed by using flow cytometry as per standard protocol (18). Briefly, SSC-15 cells at a density of $5 \times 10^{5}$ cells/well plated in 6-well and were exposed to varying concentrations $(200,250,300 \mu \mathrm{M})$ of BBR in a $5 \%$ humidified $\mathrm{CO}_{2}$ incubator at $37^{\circ} \mathrm{C}$ for $24 \mathrm{~h}$. The SSC-15 cells were harvested, washed with pre-cold PBS twice, and were resuspended in binding buffer at a density of $1 \times 10^{5}$ cells $/ \mathrm{mL}$. After resuspension, cells were incubated with $5 \mu \mathrm{L}$ each of FITC-conjugated annexin $\mathrm{V}$ and PI, followed by incubated in dark for $15 \mathrm{~min}$ at $4^{\circ} \mathrm{C}$. After incubation samples were analyzed for quantification of apoptosis by using flow cytometry (BD FACS Calibur; BD Bioscience)

\section{Reverse transcription-quantitative polymerase chain reaction (RT-qPCR)}

RNA extraction was performed by using the TRIzol RNA isolation kit as described previously (19), Briefly, SSC-15 cells at a density of $0.5 \times 10^{6}$ were plated in a 6-well plate. After adhering to the bottom surface of well, SCC-15 were exposed to varying concentrations of BBR $(200,250,300 \mu \mathrm{M})$ in a 6-well plate for $24 \mathrm{~h}$. Using the PrimScript RT reagent kit, extracted RNA was converted to cDNA by reverse transcription. After cDNA synthesis, QPCR was achieved by using the QuantiTect SYBR-Green PCR Kit, on a sequencer. The PCR conditions were setup is $95^{\circ} \mathrm{C}$ for five min, followed by more than 35 cycles with incubation conditions $95^{\circ} \mathrm{C}$ for $30 \mathrm{sec}, 58^{\circ} \mathrm{C}$ for $30 \mathrm{sec}$, and $72^{\circ} \mathrm{C}$ for 30 sec. The primers used for miR-21 (forward 5'AAATTGGGAGGACTCCAAGC-3', reverse 5'GAAGGAAAAAGTATGTCAGTGCAA-3'), miR155 (forward 5'-GAATAATATATTTCCTTGGTTTGGAA-3', reverse 5'-TTCAAAAGAAACGTATGAAGTAAAA-3') and U6 (forward 5'GCTTCGGCAGCACATATACTAAAAT-3', Reverse 5'-CGCTTCACGAATTTGCGTGTCAT3') stem-loop RT-qPCR are presented in table. The relative gene expressions were analyzed by employing the $2-\Delta \Delta \mathrm{CT}$ method, and internal control as U6.

\section{Statistical analysis}

All the evaluated experiments were accomplished equal to or more than three independent times. The latest version of software GraphPad Prism was used for statistical analysis of all independent unbiased experiments. The results obtained were denoted as the mean of \pm SEM. Entire results were calculated and processed One-way ANOVA, wherein a $p$-value not more than 0.05 was reflected as significant (*means $\mathrm{p}<0.05$. $* * \mathrm{p}<0.01$, and $* * *$ $\mathrm{p}<0.001)$.

\section{RESULTS}

BBR prevents cell proliferation and clonogenic property of HNSC cells

In recent studies, various reports showed that BBR exhibits an antiproliferative effect on numer- 
ous cancer cell lines. To evaluate the antiproliferative effect of BBR, the cell viability was analyzed by using an MTT assay. Following the treatment to varying concentrations $(200,250,300 \mu \mathrm{M})$ of BBR for $24 \mathrm{~h}$. We found BBR significantly reduces the percentage of viable cells of HNSC cell lines (SSC15 and SSC-4) in a dose-dependent manner. However, when calculated the $\mathrm{IC}_{50}$ values of $\mathrm{BBR}$ against all the HNSC cell lines used in the experiment by using GraphPad Prism software. The observed $\mathrm{IC}_{50}$ values are 235 and $242 \mu \mathrm{M}$ for SSC-4 and SSC-15, respectively. However, against normal transformed cell line (NBL-4), BBR kills $50 \%$ cells at $9450 \mu \mathrm{M}$, indicated that HNSC cells are more sensitive to BBR, in contrast to normal transformed cells (Fig. 1A).

In addition to antiproliferative activity, we also intended to evaluate, whether BBR could inhibit clonogenic property of HNSC cells. Our results demonstrated that the colony formation ability of
HNSC cells was inhibited by BBR treatment. Interestingly, we found, sub-toxic doses of BBR $(100,150,200$, and $250 \mu \mathrm{M})$ significantly attenuate the clonogenic property of SSC-15 cells when compared to untreated cells and positive control staurosporine (Figs. 1B and 1C). Collectively, these results revealed that BBR inhibits cancer cell proliferation and clonogenic property of HNSC cells specifically with a safe toxicity profile against transformed normal cells.

\section{BBR induces autophagy in HNSC cells}

Numerous reports suggest that BBR induces autophagy and kills tumor cells by various mechanisms. Therefore, we intended to seek whether BBR could also promote autophagy in HNSC cells. To do this, SSC-15 cells were plated in 6-well, after adhered to the bottom surface of well, cells were treated with varying doses of BBR for $24 \mathrm{~h}$. After treating SSC-15 cells for $24 \mathrm{~h}$, immunoblotting
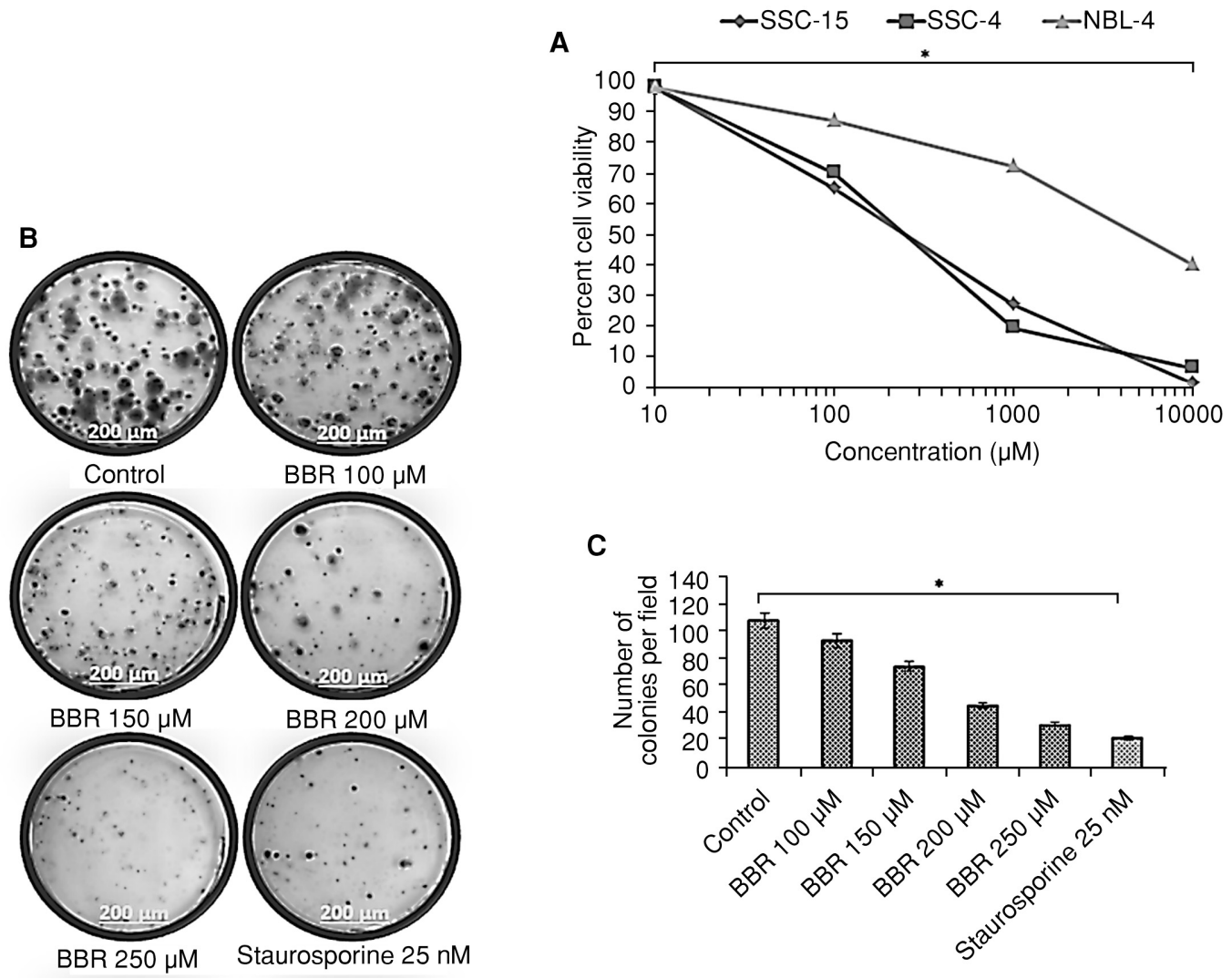

C

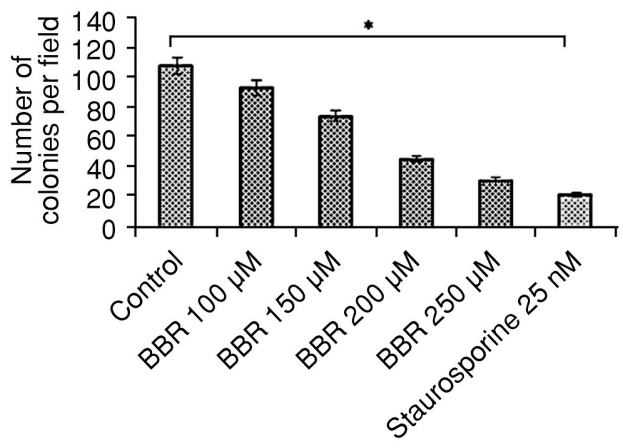

Figure 1. BBR inhibits cell proliferation and clonogenic properties of HNSC cells. (A) The effect of BBR on the cell viability of HNSC (SSC-15, SSC-4, and transformed normal cell line NBL-4) was determined by the MTT assay. (B) SSC-15 cells were treated with BBR in a concentration-dependent manner along with positive control staurosporine and untreated control for $24 \mathrm{~h}$, to determine the anti-clonogenic property of BBR. Scale bar: $200 \mu \mathrm{m}$; 10x. (C) Bar diagram showing quantification of a number of colonies of SSC-15 cells per field. The data represent the mean value $\pm \mathrm{SE}$ of three independent experiments * $\mathrm{p}<0.05$. 

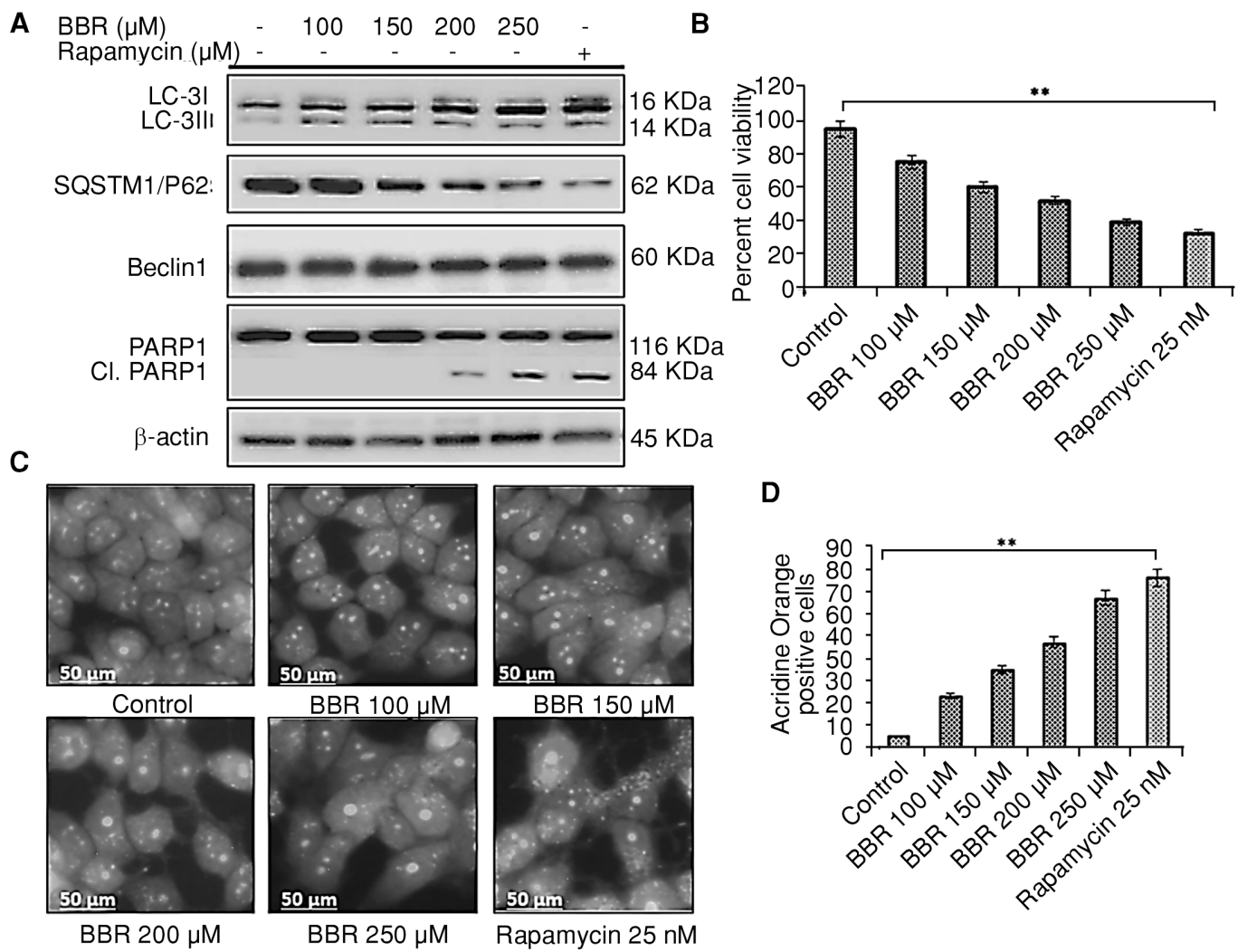

Figure 2. BBR induces autophagy and augments cell death in HNSC cells. (A) Immunoblotting analysis of HNSC cells (SSC-15) exposed to various BBR concentrations along with untreated control and positive control rapamycin $25 \mu \mathrm{M}$ shows the LC-3I to LC-3-II conversion, p62 SQSTMI expression Beclin1 expression and PARP1 along $\beta$-actin as an internal control. (B) Bar diagram showing the percentage of viable cells after treating SSC-15 in a dose-dependent manner with BBR. (C) Representative microphotographs of HNSC cells (SSC-15) displays AVOs due to acridine orange staining analyzed by fluorescent microscopy, exposed to indicate the concentration of BBR along with untreated control and positive control rapamycin $25 \mu \mathrm{M}$ to evaluate the phenotypic characteristics of autophagy. Scale bar: $50 \mu \mathrm{m}$; 10x. (D) The bar diagram presents the quantification of AO-positive cells analyzed by fluorescent microscopy in the above experiment. Three independent and random fields representing 100 cells were counted. The data represent the mean value \pm SE of 3 independent experiments. $* * \mathrm{p}<0.01$

results revealed a prominent conversation of microtubule-associated protein 1light chain $3 \beta$-I (LC-3I) to microtubule-associated protein 1light chain $3 \beta$-II (LC-3II), which is a distinctive hallmark of autophagosome maturation. To check whether BBR treatment could affect the autophagy flux in HNSC cells, we found a drastic reduction in the expression of sequestrosome 1 protein p62 with decreased band intensity in lanes exposed to a higher concentration of BBR. Interestingly, we also observed prominent PARP1 cleavage at higher concentrations of BBR. However, the protein expression of another important sensor of the autophagy process, beclin 1 did not change and remains consistently unchanged (Fig 2A). Additionally, In HNSC cells, BBR inhibited cell proliferation by inducing autophagy-mediated cell death in a dose-dependent manner (Fig. 2B).
Further, the study was commenced to inspect autophagic cells by acridine orange (AO) staining. To do this, SSC-15 cell was seeded in a 6-well plate and exposed to varying concentrations of BBR. We observe prominent visualization of AVOs appeared bright red in the cytoplasm of SSC-15 cells exposed to higher concentrations of BBR treatment (Figs. 2C and 2D). Collectively, these results suggest that BBR treatment stimulates autophagic flux and augments autophagic cell death in HNSC cells.

\section{BBR promotes apoptotic cell death in HNSC cells} BBR has been shown to promote apoptosis by activating caspases, through cytochrome c release from mitochondria (intrinsic apoptosis), AMPK activation, and induction of reactive oxygen species (ROS). Therefore, we intended to seek whether 
BBR could also promote apoptotic cell death in HNSC cells. After treating SSC-15 cells to varying concentrations of BBR $(100,150,200$, and $250 \mu \mathrm{M})$ for $24 \mathrm{~h}$, SSC-15 cells were incubated with PI and annexin V staining. Our flow-cytometry analysis revealed that a significant population of cells undergoes apoptosis $(27.1 \%)$ at a higher concentration of BBR $(250 \mu \mathrm{M})$ when compared to untreated control $(6.6 \%)$ and positive control staurosporine $25 \mathrm{nM}$ (33.7\%) (Figs. 3A and 3B). To further confirm the activation of apoptosis we perform immunoblotting after treating SSC-15 cells with BBR in a dosedependent manner. We observe prominent cleavage of PARP1 and caspase- 3 proteins in the lanes where cells were exposed to higher concentrations of BBR compared to the control cell line (Fig. 3C). Together, these results demonstrate that BBR induces potent antiproliferative by pushing cells towards apoptosis in a dose-dependent manner

\section{BBR downregulates miR-155 in HNSC cells}

The potential application of miRNAs in cancer therapy has been demonstrated in many studies. miRNA's function either as a tumor suppressor or oncogene. Tumor suppressor miRNAs are downregulated in cancers, whereas oncogene miRNAs are up-regulated. Previous reports revealed that BBR modulates the miRNA expression in various cellular models. Therefore, we also wanted to evaluate whether BBR could modulate the expression of miRNAs in HNSC cells. The current study evaluates the effect of BBR on the expression of miRNAs. After the exposure of HNSC cells with varying doses of BBR for $24 \mathrm{~h}$ along with control DMSO. The total RNA was isolated to analyze miRNAs by using RT-qPCR. Our results demonstrated that significant upregulation of miR-155 and downregulation of miR-21 was observed after SSC-15 cells were exposed to BBR in a dose-dependent manner when compared with untreated control (Figs. 4A and 4B) (Table 1). Collectively, these results revealed that higher doses of BBR treatment downregulates miR-21 and upregulates miR-155. This modulation of tumor suppressor and oncogenic miRNAs could sensitize head and neck tumor cells thereby exert antiproliferative effect and induces simultaneous induction of autophagy and apoptosis in HNSC cells.

\section{A}

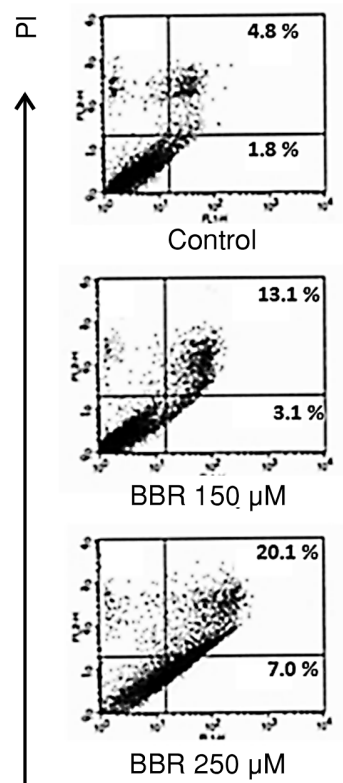

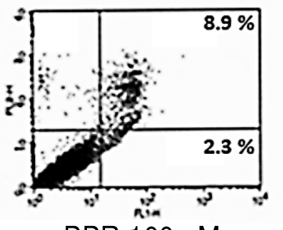
BBR $100 \mu \mathrm{M}$

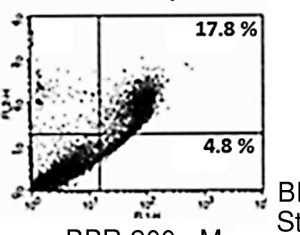
BBR $200 \mu \mathrm{M}$

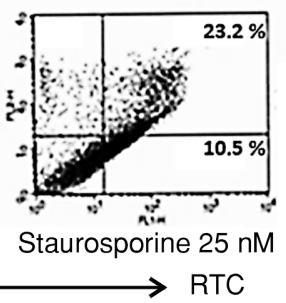

\section{B}

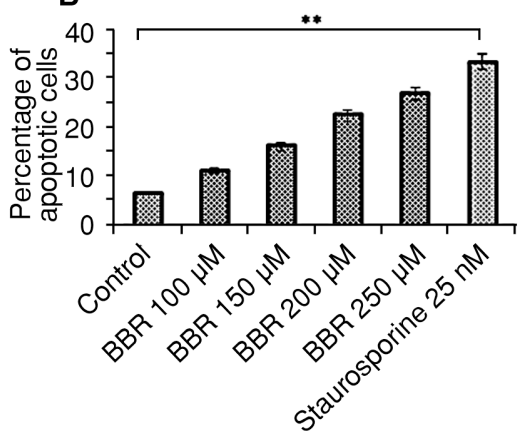

D

BR $(\mu \mathrm{M})$

- $100 \quad 150 \quad 200 \quad 250$.

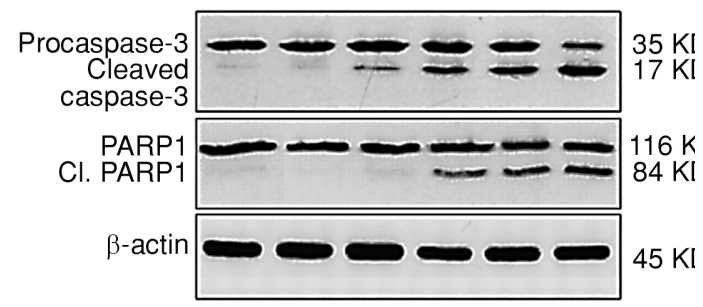

Figure 3. BBR induces apoptosis in HNSC cells. A. HNSC cells (SSC-15) were exposed to indicated concentrations of BBR along with untreated control and positive control staurosporine $25 \mathrm{nM}$ for $24 \mathrm{~h}$ and then subjected to Annexin V FITC and propidium iodide staining and analyzed by FACS. (B) Bar diagram showing quantification of Annexin V FITC positive cells analyzed by FACS. (C) Immunoblotting analysis showing prominent PARP cleavage in lanes where cells were exposed to higher concentrations of BBR and staurosporine $25 \mathrm{nM}$ treatment and internal control $\beta$-actin. The data represent the mean value \pm SE of three independent experiments $* * \mathrm{p}<0.01$. 
A

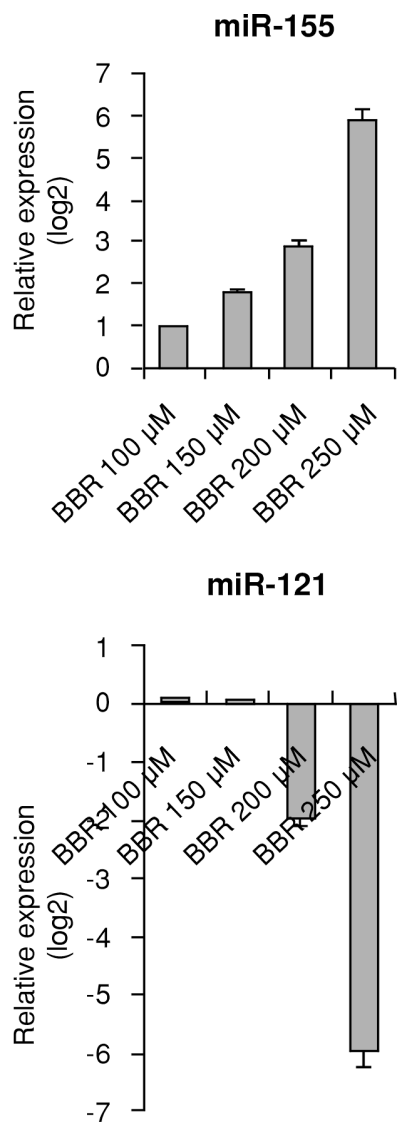

Figure 4. BBR modulates miR-155 and miR-21 expression profile in SSC-15 cells. (A) The bar diagram represents relative fold change in miR-155 expression in log 2 after SSC-15 cells were exposed to varying concentrations of BBR for $24 \mathrm{~h}$. (B) The bar diagram represents relative fold change in miR-21 expression in $\log 2$ after SSC- 15 cells were exposed to varying concentrations of BBR for $24 \mathrm{~h}$. The data represent the mean value $\pm \mathrm{SE}$ for the fold change in the expression of miRNA in log 2 scale between BBRtreated SSC-15 cells and untreated SSC-15 cells obtained from three independent experiments.

\section{DISCUSSION}

The major oncogenic drivers in HNSC are high intake of alcohol, long-term use of tobacco, and high-risk infections-associated Human Papilloma Virus. The high prevalence and increased mortality rate in HNSC reduce life expectancy and quality of life (20). The therapeutic options for HNC are surgery followed by radiotherapy and chemotherapy (21). Although significant advancement has been achieved in medical intervention, which includes surgical intervention followed by radiation therapy and chemotherapeutics. However, the five-year survival period in HNSC patients is still disheartening from the last 20 years (22). Although chemotherapy is believed to be one of the major medical intervention to deal HNC, however, the effectiveness and adverse effects of clinical chemotherapeutic drugs against HNSC is dismal (23). To manage and increase the quality of life of HNC patients, there is a need for the development of novel drugs that can kill tumor cells specifically with the least undesirable effects on normal cells. (24). Thus, the primary purpose of the development of chemotherapeutic drugs is focused on the killing of cancer cells specifically and leaving normal cells unaffected (25). Natural product compounds from a variety of sources are used to treat various ailments since ancient times. Natural compounds and their associated structural moieties play an important part in the development of drug discovery (26). In the recent past, strategies have been developed to evaluate the safety profile and drug efficacy of plant-based natural compounds in combination with clinically approved drugs or alone for the development of effective chemothera-

Table 1. Modulation of miRNA expression (miR-155, and miR-21) after SSC-15 cells were exposed to a dosedependent concentration of BBR.

\begin{tabular}{|c|c|c|c|}
\hline $\begin{array}{c}\text { BBR treatment } \\
(\mu \mathrm{M} ; 24 \mathrm{~h}\end{array}$ & $\begin{array}{c}\text { miRNA-155 expression } \\
\text { after normalized with control }\end{array}$ & $p$-value & $\begin{array}{c}\text { Up-/down } \\
\text { regulation }\end{array}$ \\
\hline 100 & 0.95 & 0.034 & up \\
\hline 150 & 1.78 & 0.045 & up \\
\hline 200 & 2.86 & 0.039 & up \\
\hline 250 & 5.87 & 0.0012 & up \\
\hline $\begin{array}{c}\text { BBR treatment } \\
(\mu \mathrm{M} ; 24 \mathrm{~h}\end{array}$ & $\begin{array}{c}\text { miRNA-21 expression } \\
\text { after normalized with control }\end{array}$ & $p$-value & $\begin{array}{c}\text { Up-/down } \\
\text { regulation }\end{array}$ \\
\hline 100 & 0.053 & 0.014 & up \\
\hline 150 & 0.034 & 0.034 & up \\
\hline 200 & -1.98 & 0.029 & down \\
\hline 250 & -5.98 & 0.019 & down \\
\hline
\end{tabular}


peutic drugs (27). Among the natural products, $\mathrm{BBR}$ is an iso-quinoline type of alkaloid extracted from Rhizoma coptidis (28) and exerts a potent antiproliferative effect on a wide variety of tumor cell models (29). Recent pharmacology studies of BBR have demonstrated that BBR exerts potential anticancer activity, by inducing autophagy, apoptosis, and modulating miRNAs (30).

The current study demonstrates BBR exerts significant cytotoxic activity in HNSC cells and attenuates the clonogenic property of SSC-15 cells in a dose-dependent manner. Mechanistically, BBR simultaneously induces autophagy as well as apoptosis-mediated cell death in HNSC cells. Furthermore, BBR-mediated autophagy was observed by a study conversion of LC-3I to LC-3II with a concomitant reduction in autophagy substrate protein sequestrosome (p62) in a dose-dependent manner. Moreover, BBR activated the apoptotic signaling pathway by up-regulating and cleaving proapoptotic proteins PARP1 and caspase-3 in HNSC cells at higher concentrations of BBR. Additionally, we demonstrate that BBR modulates miRNAs, by upregulating tumor suppressor miR-155 and downregulating the oncogenic miR-21 expression in SSC15 cells in a dose-dependent manner.

Despite having other pharmacological activities, BBR has gained tremendous attention as a promising chemotherapeutic agent $(31,32)$. BBR exerts anti-tumor activity, inhibits migration, and induces apoptosis in a wide variety of cancer cell lines (32). BBR modulates and interacts with a plethora of molecules that play an important role in regulating tumorigenesis (33). Recent reports revealed that $\mathrm{BBR}$ exerts antiproliferative effect by inducing mitochondrial-dependent cell death in breast carcinoma cells by reducing the protein expression of anti-apoptotic protein $\mathrm{Bcl}-2$ and upregulates tumor suppressor miR-21-3p expression in hepatocellular carcinoma (32). Consistent with previous reports, we notice that BBR treatment exerts significant antiproliferative effect with $\mathrm{IC}_{50}$ in a range of $235-242 \mu \mathrm{M}$ and inhibits clonogenic property of HNSC cells in a dose-dependent manner.

Autophagy is a catabolic process in which cell utilizes damaged organelles or macromolecules thereby generates recycled metabolic energy for regulating cellular homeostasis especially during stress conditions (34). Besides apoptosis, natural compounds have been reported to induce autophagic cell death (programmed cell death type II) in cancer cells (35). The previous report also revealed that BBR suppresses hepatocellular carcinoma cells, by simul- taneous induction of mitochondrial-mediated apoptosis and autophagic cell death in hepatocellular carcinoma cells (36). Additionally, various BBR derivatives have been reported to induce autophagy in colorectal cancer cells (37). Consistent with the previous studies, we observe BBR augments autophagy by visualizing prominent orange-red color cytoplasmic acidic autophagosomes stained with acridine orange. Further, we observe study conversion of autophagy protein LC-3I to LC-3II with concomitant reduction of autophagy flux protein sequestrosome (P62) when SSC-15 cells were exposed to a dose-dependent concentration of BBR. The common mechanism of inducing cell death by natural chemopreventive agents is programmed cell death type I called apoptosis (38). The activation of death receptor-mediated apoptosis called extrinsic apoptosis pathway or the intrinsic apoptosis pathway which is the mitochondria-dependent pathway, operated when there are mitochondrial membrane depolarization and cytochrome c liberation from mitochondria (39). Previous reports in colorectal cancer and hepatocellular carcinoma revealed that BBR induces caspase-3 and PARP1 mediated apoptosis (40). Additionally, BBR mediated depolarization of mitochondrial membrane potential releases cytochrome C. This results in elevation of ROS level which activates PARP1 mediated apoptosis (41). Consistent with the previous reports, our results revealed that a significant population of apoptotic cells $(27.1 \%)$ was quantified by flow cytometry when SSC-15 cells were exposed to a higher dose $(250 \mu \mathrm{M})$ of BBR. Additionally, our immunoblotting results also support that BBR activates and cleaves proapoptotic proteins procaspase-3 and PARP1 in SSC-15 cells in a dose-dependent manner.

Recent advancements in genomics confirmed that miRNAs are the key players in the progression of tumorigenesis (42). The modulation of miRNAs by antitumor agents plays a crucial role in cancer diagnostics and therapeutics (43). In the recent past, BBR has been reported to inhibit cell proliferation and induces apoptosis in HCC by upregulating miR-21$3 p$ (44). Additionally, BBR sensitizes cisplatin-resistant ovarian cancer cells by downregulates miR-21 (45). Consistent with the previous studies, our results demonstrate that BBR modulates miRNA expression of SSC-15 cells in a dose-dependent manner. The RT-qPCR analysis revealed that BBR modulates miRNA expression by downregulating and upregulating the expression of miR-21 and miR-155 respectively, which play a key role in tumorigenesis and apoptosis mechanisms of head and neck cancer. Collectively, these findings reveal that BBR modu- 
lates biological activities by regulating the expressions of miR-155, miR-21, and sensitize malignant cells to cell death mode (autophagy and apoptosis).

\section{CONCLUSION}

Our results first time demonstrate that BBR exerts antiproliferative effect and abrogates the clonogenic property of HNSC cells. Mechanistically, BBR induces autophagy by prominent visualization of cytoplasmic orange-red color acidic autophagosomes and simultaneous conversion of autophagy protein LC-3I to LC-3II with concomitant reduction of sequestrosome protein $\mathrm{P} 62$. Moreover, BBR induces apoptosis by activates proapoptotic proteins procaspase-3 and PARP1 mediated cell killing in HNSC cells. Additionally, downmodulates and upregulates the expression of miR-21 miR-155, respectively. Collectively these results demonstrate that $\mathrm{BBR}$ is a promising anticancer therapeutic agent against HNSC cell models and could be used in future therapeutics for the chemoprevention of HNC.

\section{Acknowledgments}

The authors would like to thank the Department of Neurosurgery, Tianjin Huanhu Hospital, No. 6 Jizhao Road, Jinnan District, Tianjin, China, 300060 for grant and support.

\section{Authors contributions}

Binbin Zhang: Conceptualization, Visualization Data curation, acquisition, Formal analysis. Jingchuan He: Conceptualization, Writing, review \& editing. Kai Xue: Conceptualization, design experiments, writing review, and editing the original draft.

\section{Conflict of interests}

The authors declare no conflict of interest.

\section{REFERENCES}

1. Bray F., Ferlay J., Soerjomataram I., Siegel R.L., Torre L.A., Jemal A.: CA Cancer J. Clin. 68, 394 (2018).

2. Holmes B.J., Westra W.H.: Diagn. Cytopathol. 42, 85 (2014).

3. Vermorken J.B., Mesia R., Rivera F., Remenar E., Kawecki A., et al.: New Eng. J. Med. 359, 1116 (2008).
4. O'driscoll L.: Anticancer Res. 26, 4271 (2006).

5. Ha T.-Y.: Immune Netw. 11, 135 (2011).

6. Dalmay T., Edwards D.: Oncogene 25, 6170 (2006).

7. Xu Y., Xia F., Ma L., Shan J., Shen J., et al.: Cancer lett. 310, 160 (2011).

8. Diab S., Diab D., Saab A.M., Gambari R., Tannoury M., et al.: European J. Biomed. Pharm. Sci. 4, 876 (2017).

9. Liu B., Wang G., Jie Yang J., Xuediao Pan X., Yang Z., et al.: PloS One 6, 6 pages (2011).

10. McCubrey J.A., Lertpiriyapong K., Steelman L.S., Abrams S.L., Li V Yang L.V., et al.: Aging (Albany NY) 9, 1477 (2017).

11. Notte A., Leclere L., Michiels C.: Biochem. Pharmacol. 82, 427 (2011).

12. Yu R., Zhang Z.-Q., Wang B., Jiang H.-X., Cheng L., Shen L.-M.: Cancer Cell Int. 14, 49 (2014).

13. Rah B., Ur Rasool R., Nayak D., Yousuf S.K., Mukherjee D., et al.: Autophagy 11, 314 (2015).

14. Shakeel-u-Rehman, Rah B., Lone S.H., Rasool R.U., Farooq S., et al.: J. Med. Chem. 58, 3432 (2015)

15. Rah B., Amin H., Yousuf K., Khan S., Jamwal G., et al.: PloS One 7, e44039 (2012).

16. Sinha S., Mishra P., Amin H., Rah B., Nayak D., et al.: Eur. J Med. Chem. 60, 490 (2013).

17. Alnuqaydan A.M., Rah B., Almutary A.G., Chauhan S.S.: Am. J. Cancer Res. 10, 799 (2020).

18. Rasool R.U., NayakD., Chakraborty S., Faheem M.M., Rah B., et al.: Oncogenesis 6, e341 (2017).

19. He R.Q., Yang X., Liang L., Chen G., Ma J.: Oncol. Lett. 15, 5517 (2018).

20. Dirks S.J., Monopoli M.: in Healthy Aging, pp. 213-226, Springer, 2019.

21. Strojan P., Ferlito A., Langendijk J.A., Corry J., Woolgar J.W., et al.: Head Neck 35, 286 (2013).

22. Wu P.C., Posner M.C.: Lancet Oncol. 4, 481 (2003).

23. Corvò R.: Radiother. Oncol. 85, 156 (2007).

24. Forastiere A., Koch W., Trotti A., Sidransky D.: N. Engl. J. Med. 345, 1890 (2001).

25. Lebedeva I.V., Su Z.-Z., Sarkar D., Fisher P.B.: in Seminars in cancer biology, Vol. 13, pp. 169178, Elsevier, 2003.

26. Chen J., Li W., Yao H., Xu J.: Fitoterapia 103, 231 (2015).

27. Roy A., Jauhari N., Bharadvaja N.: in Anticancer Plants: Natural Products and Biotechnological Implements. Akhtar M.S., 
Mallappa Swamy K., Eds., Vol. 2, pp. 109-139, Springer, 2018.

28. Sun Y., Xun K., Wang Y., Chen X.: AntiCancer Drugs 20, 757 (2009).

29. Okubo S., Uto T., Goto A., Tanaka H., Nishioku T., et al.: Am. J. Chin. Med. 45, 1497 (2017).

30. Ayati S.H., Fazeli B., Momtazi-Borojeni A.A., Cicero A.F.G., Pirro M., Sahebkar A.: Crit. Rev. Oncol. Hemat. 116, 147 (2017).

31. Vuddanda P.R., Chakraborty S., Singh S.: Expert Opin. Investig. Drugs 19, 1297 (2010).

32. Guamán Ortiz L.M., Lombardi P., Tillhon M., Scovassi A.I.: Molecules 19, 12349 (2014).

33. Wang N., Tan H.-Y., Li L., Yuen M.-F., Feng Y.: J. Ethnopharmacol. 176, 35 (2015).

34. Wu W.K.K., Coffelt S.B., Cho C.H., Wang X.J., Lee C.W., et al.: Oncogene 31, 939 (2012).

35. Wong V.K.W., Li T., B.Y.K., Ma E.D.L., Yip N.C., et al.: Cell Death Dis. 4, e720 (2013).
36. Wang N., Feng Y., Zhu Y., Tsang C.-M., Man K., et al.: J. Cell. Biochem. 111, 1426 (2010).

37. Grycova L., Dostál J., Marek R.: Phytochemistry 68, 150 (2007).

38. Safarzadeh E., Shotorbani S.S., Baradaran B.: Adv. Pharm. Bull. 4, 421 (2014).

39. Robertson G.S., LaCasse E.C., Holcik M.: in Pharmacology, pp. 455-473, Elsevier, 2009.

40. Wang L., Liu L., Shi Y., Cao H., Chaturvedi R., et al.: PloS One 7, 12 pages (2012).

41. Lin C.-C., Yang J.-S., Chen J.-T., Fan S., FuShun Yu, et al.: Anticancer Res. 27, 3371 (2007).

42. Mirnezami A., Pickard K., Zhang L., Primrose J., Packham G.: Eur. J. Surg. Oncol. 35, 339347 (2009).

43. Blower P.E., Chung J.-H., Verducci J.S., Lin S., Park J.-K., et al.: Mol. Cancer Ther. 7, 1 (2008).

44. Lo T.-F., Tsai W.-C., Chen S.-T.: PLoS One 8, 9 pages (2013).

45. Liu S., Fang Y, Shen H., Xu W., Li H.: Acta Biochim. Biophys. Sin. 45, 756 (2013).

(C) 2020 by Polish Pharmaceutical Society. This is an open-access article under the CC BY NC license (c) (1) (9) (http://creativecommons.org/licenses/by-nc/4.0/). 